\title{
Multi-Sensor Kernel Design for Time-Frequency Analysis of Sparsely Sampled Nonstationary Signals
}

\author{
Yimin D. Zhang ${ }^{\dagger}$, Liang Guo ${ }^{\ddagger \dagger}$, Qisong $\mathrm{Wu}^{\dagger}$, and Moeness G. Amin ${ }^{\dagger}$ \\ $\dagger$ Center for Advanced Communications, Villanova University, Villanova, PA 19085, USA \\ $\ddagger$ School of Physics and Optoelectronic Engineering, Xidian University, Xi'an 710071, China
}

\begin{abstract}
In this paper, we examine the sparsity-based timefrequency signal representation (TFSR) of randomly thinned nonstationary signals in a multi-sensor platform to yield improved performance with reduced number of samples in each sensor. The property that different sensors share identical autoterm time-frequency regions renders the TFSR a group sparse reconstruction problem, which is effectively solved using the compressive sensing techniques for high-fidelity TFSR reconstruction. We exploit the adaptive optimal kernel (AOK) to effectively preserve signal auto-terms and mitigate cross-terms. High level of noise and artifacts due to missing samples, however, may render AOK ineffective if designed for each sensor separately. We develop a robust multi-sensor AOK design based on data fusion across all sensors so as to enhance the signal auto-terms while effectively mitigating artifacts, cross-terms, and noise. The superior performance of the proposed multi-sensor AOK design is demonstrated through the comparison with its single-antenna counterpart and data-independent kernels.
\end{abstract}

Index Terms-Time-frequency analysis, array processing, compressive sensing, missing data sample, sparse reconstruction

\section{INTRODUCTION}

A large class of nonstationary signals, particularly those characterized by their instantaneous frequencies (IFs), are often encountered in practice, including radar, sonar, communications and biomedical applications [1-6]. In particular, by exploiting multiple sensors, array processing of nonstationary signals finds broad applications, such as direction finding, source separation, jammer suppression, and source localization [7-14].

For such nonstationary signals, it is advantageous to represent them in the joint time-frequency (TF) domain to exploit signal sparsity and reveal the local signal behaviors. Reduced interference distributions (RIDs) are well-known bilinear timefrequency signal representation (TFSR) methods for nonstationary signals to mitigate the effect of undesirable crossterms. These distributions can be conveniently defined through the use of proper kernel in ambiguity functions (AFs) [1519]. Traditional TF analysis assumes that the analyzed signals are uniformly sampled with the rate equal to or higher than the Nyquist rate. In many real-world applications, however, nonstationary signals are often observed with missing samples due to fading, obstruction, and/or impulsive noise [20], thus yielding artifacts that are spread in the entire TF domain.

The work of L. Guo was supported in part by the National Natural Science Foundation of China (Grant No.61107006), by the Fundamental Research Funds for the Central Universities (Grant No.K5051305005 and No.NSIY171412) and by China Scholarship Council.
These artifacts can be mitigated by applying TF smoothing kernels [21]. In multi-sensor systems, artifact mitigation can also be accomplished by averaging $\mathrm{AF}$ with respect to the sensors [10, 22].

In this paper, inspired by the sparse reconstruction of TFSR based on the sparsity or group sparsity [22-24], we examine the sparsity-based TFSR reconstruction of randomly thinned nonstationary signals in a multi-sensor platform to yield improved performance with reduced number of samples in each sensor. In particular, we modify the well-known datadependent TF kernel, adaptive optimal kernel (AOK), to the multi-sensor situation for robust TFSR reconstruction with suppressed artifacts induced by the missing data. To achieve this objective, the spatial averaging operation becomes an integral part of the overall design paradigm. That is, the optimization or kernel parameters is performed at the averaged AF overall sensors, instead of the AF obtained in each individual sensor. Once the kernel is designed, the corresponding TF signal representation can be obtained through the use of Cohen's class, i.e., using two-dimensional (2-D) Fourier transform of the kernelled AF. Furthermore, by replacing the Fourier transform with sparse parametric approach, the compressive sensing (CS) technique achieves enhanced resolution in the TF domain. In particular, by utilizing the one-dimensional (1-D) Fourier transform relationship between the instantaneous autocorrelation function (IAF) and the TF distribution, rather than the 2-D Fourier transform relationship between the AF and TF domains, CS reconstruction not only reduces the computation complexity, but also improves the performance as a result of enabling the consideration of local sparsity over each time instant.

Notations. We use lower-case (upper-case) bold characters to denote vectors (matrices). $\mathcal{F}_{x}$ and $\mathcal{F}_{x}^{-1}$ respectively represent the discrete Fourier Transform (DFT) and inverse DFT (IDFT) with respect to $x$. In particular, $\mathbf{I}_{N}$ denotes the $N \times N$ identity matrix. $(\cdot)^{T}$ and $(\cdot)^{*}$ respectively denote the transpose and complex conjugates of a matrix or vector. In addition, $\mathbb{C}^{N \times K}$ denotes the complete set of $N \times K$ complex entries.

\section{Signal MODEL}

Consider $K$ narrowband nonstationary signals impinging on an array consisting of $N$ sensors, where $K<N$ is assumed. The $N \times 1$ received data vector $\mathbf{y}(t)$ and the $K \times 1$ source signal vector $\mathbf{d}(t)$ are related by

$$
\mathbf{y}(t)=\mathbf{H d}(t)+\mathbf{n}(t)
$$


where $t \in[1, \cdots, T]$ is the time index, $\mathbf{H}=$ $\left[\mathbf{h}_{1}, \mathbf{h}_{2}, \cdots, \mathbf{h}_{K}\right] \in \mathbb{C}^{N \times K}$ is the mixing matrix with $\mathbf{h}_{q}$ denoting the spatial signature of the $q$-th source. $\mathbf{n}(t) \in \mathbb{C}^{N \times 1}$ is an additive noise vector that consists of independent and identically distributed (i.i.d.) zero-mean, white and complex Gaussian distributed processes with variance $\sigma_{n}^{2} \mathbf{I}_{N}$. The noise elements are assumed to be independent of the signals, which are assumed to be deterministic. Here, each element of $\mathbf{d}(t)=\left[d_{1}(t), d_{2}(t), \cdots, d_{K}(t)\right]^{T}$ is a single component frequency modulated (FM) signal and arrives with or without an angular bearing. Consider the thinned sampling of the array observations with a random pattern applied to each array sensor, where the number of missing samples is $M_{q}$ satisfying $M_{q}<T$ for $q=1, \cdots, N$. As such, for the $q$-th array sensor, the thinned observation, $x_{q}(t)$, can be expressed as the product of $y_{q}(t)$, expressed in (1), and the following observation mask,

$$
b_{q}(t)= \begin{cases}1, & \text { if } t \in \mathbb{S}_{q} \\ 0, & \text { if } t \notin \mathbb{S}_{q}\end{cases}
$$

where $\mathbb{S}_{q} \subset\{1, \cdots, T\}$ is the set of observed time instants and its cardinality is $\left|\mathbb{S}_{q}\right|=T-M_{q}$. For simplicity but without loss of generality, we assume that $M_{q}$ to be the same for all sensors, i.e., $M_{q}=M$, for $q=1, \cdots, N$, whereas $b_{q}(t)$ may or may not be the same.

\section{TIME-FREQUENCY DISTRIBUTION WITH MODIFIED ADAPTIVE OPTIMAL-KERNEL}

\section{A. Adaptive Optimal Kernel}

In the rectangular form, the $\mathrm{AF}$ for signal $x(t)$ is defined as:

$$
A(\theta, \tau)=\int_{-\infty}^{\infty} x\left(t+\frac{\tau}{2}\right) x^{*}\left(t-\frac{\tau}{2}\right) e^{-j 2 \pi \theta t} d t
$$

where $\theta$ and $\tau$, respectively, denote the frequency shift and the time lag. To reduce the effect of cross-terms, which lie away from the origin in the ambiguity domain, a TF kernel function of low-pass filter characteristics is often applied to the AF.

Time-frequency kernels can be data-independent or datadependent. The latter often yields better performance due to its adaptivity to the signal. A well-known data-dependent kernel is the AOK, which is obtained by solving the following optimization problem [17]:

$$
\begin{array}{r}
\max _{\Phi} \int_{0}^{2 \pi} \int_{0}^{\infty}|A(r, \psi) \Phi(r, \psi)|^{2} r d r d \psi \\
\text { subject to } \Phi(r, \psi)=\exp \left(-\frac{r^{2}}{2 \sigma(\psi)}\right) \\
\frac{1}{4 \pi^{2}} \int_{0}^{2 \pi} \int_{0}^{\infty}|\Phi(r, \psi)|^{2} r d r d \psi \\
=\frac{1}{4 \pi^{2}} \int_{0}^{2 \pi} \sigma(\psi) d \psi \leq \alpha,
\end{array}
$$

where $\alpha \geq 0$. Note that in the above expression the AF and the kernel function are defined in terms of the radius $r$ and the aspect angle $\psi$. The AF in the polar coordinates can be obtained through direct calculation of polar samples or by interpolation from its rectangular form.

\section{B. Multi-sensor Adaptive Optimal Kernel}

The auto-terms TFDs are real and also positive for meaningful TF points where the signal energy is concentrated. On the other hand, the values and signs of the cross-terms between different signal components depend on the relative phase between the contributing signals. This phase changes across the antenna array due to respective propagation delays. As such, the simple averaging of TFDs over different antennas disfavors the cross-terms and enhances the auto-terms. The same averaging operation reduces cross-terms if the spatial correlation between the contributing signals is low [10]. Due to the 2-D Fourier transform relationship between the AF and TFD, antenna averaging also enhances auto-terms and reduces cross-terms that viewed in the ambiguity domain. Meanwhile, the missing data samples yield artifacts that are randomly spread over the entire $\theta-\tau$ domain, and the overall variance increases as the number of missing data samples increases. For $(\theta, \tau)$ entries where $A(\theta, \tau)$ is zero or insignificant, the variance is uniformly distributed over $\theta$ but varies with $\tau$ because of the zero-padding effect [21]. When different sampling patterns are adopted in each sensor, therefore, averaging the AF over all sensors effectively reduces the contributions of artifacts induced from missing samples.

In this paper, and stemming from the above discussions, we modify the object function (4) to obtain an improved kernel for the multi-sensor platform. Let $A_{q}(r, \psi)$ denote the $\mathrm{AF}$ in the $q$ th sensor, $q=1, \ldots, N$. The averaged AF over all sensors is

$$
A_{\Sigma}(r, \psi)=\frac{1}{N} \sum_{q=1}^{N} A_{q}(r, \psi)
$$

Then, the new kernel in the multi-sensor platform is obtained by replacing $A(r, \psi)$ in (4) by $A_{\Sigma}(r, \psi)$ in (5), yielding

$$
\begin{gathered}
\max _{\Phi} \int_{0}^{2 \pi} \int_{0}^{\infty}\left|A_{\Sigma}(r, \psi) \Phi(r, \psi)\right|^{2} r d r d \psi \\
\text { subject to } \Phi(r, \psi)=\exp \left(-\frac{r^{2}}{2 \sigma(\psi)}\right) \\
\frac{1}{4 \pi^{2}} \int_{0}^{2 \pi} \int_{0}^{\infty}|\Phi(r, \psi)|^{2} r d r d \psi \\
=\frac{1}{4 \pi^{2}} \int_{0}^{2 \pi} \sigma(\psi) d \psi \leq \alpha .
\end{gathered}
$$

Since $A_{\Sigma}(r, \psi)$ is a better representation of the sum of the ambiguity functions of the individual components compared to $A_{q}(r, \psi)$, the optimization (6) enables improved and more robust TFSR.

\section{Fourier AND CS-BASEd TIME-FREQUENCY REPRESENTATION}

Upon designing, the kernel from (6) in a multi-sensor platform, the TFD can be computed as the 2-D Fourier transform of the kernelled and averaged $\mathrm{AF}, A_{\Sigma}(r, \psi) \Phi(r, \psi)$. By converting the kernelled and averaged AF to the rectangular coordinate system, and denote the result as $\tilde{A}_{\Sigma}(\theta, \tau)$, the corresponding TFD is expressed as

$$
D_{1}(t, f)=\mathcal{F}_{\theta}^{-1}\left\{\mathcal{F}_{\tau}\left[\tilde{A}_{\Sigma}(\theta, \tau)\right]\right\} .
$$


Alternatively, we can also obtain the TFSR through a sparse reconstruction from the same kernelled and averaged AF. While earlier sparse TFSR reconstructions were based on the 2-D Fourier transform relationship between the AF and the TFSR [22, 23], it is shown in [21, 24] that the 1-D Fourier transform relationship between the IAF and the TFSR yields simpler computations and enables the exploitation of local sparsity in the TF domain.

The 1-D inverse Fourier transform of $\tilde{A}_{\Sigma}(\theta, \tau)$ with respect to $\theta$ yielding the kernelled IAF,

$$
C(t, \tau)=\mathcal{F}_{\theta}^{-1}\left[\tilde{A}_{\Sigma}(\theta, \tau)\right] .
$$

Denote $\mathbf{c}^{[t]}$ as a vector that consists of all IAF entries along the $\tau$ dimension corresponding to time $t$, and $\mathbf{w}^{[t]}$ as a vector contains all the TFSR entries with respect to the frequency for the same time $t$. According to the Fourier relationship between the IAF and the TFSR, we obtain

$$
\mathbf{c}^{[t]}=\boldsymbol{\Phi}_{\mathbf{w}}{ }^{[t]}+\boldsymbol{\epsilon}^{[t]}, \quad t \in[1, \ldots, T],
$$

where $\boldsymbol{\Phi}$ is a matrix for the IDFT operation with respect to $f$. In this paper, we use the the OMP algorithm [25] to perform sparse reconstruction of the TFSR, which is repeated in each time instant. Other compressive sensing methods, such as Lasso and Bayesian compressive sensing [26-28], can also be used for effective TFSR reconstructions.

\section{Simulation Results}

Consider a 4-element uniform linear array (ULA) with a half-wavelength interelement spacing. Two nonstationary signals impinge on the array with their respective arbitrary spatial signatures. The latter is also referred to as generalized steering vectors and can be due to multipaths. The signatures are randomly generated with independent complex Gaussian distribution with zero mean and unit variance, expressed as

$$
\begin{aligned}
& \mathbf{H}^{T}= \\
& {\left[\begin{array}{rrrr}
-1.22+0.24 j & 1.39+0.66 j & 0.16-0.39 j & 0.28-0.39 j \\
1.13+0.95 j & -0.44-0.35 j & -0.50-0.26 j & 1.13-0.33 j
\end{array}\right]}
\end{aligned}
$$

The signals emitted from the two sources are polynomial FM signals with closely separated signatures. Their IF laws are expressed as [21]

$$
\begin{aligned}
& f_{1}(t)=0.05+0.1 t / T+0.1 t^{2} / T^{2} \\
& f_{2}(t)=0.15+0.1 t / T+0.1 t^{2} / T^{2}
\end{aligned}
$$

where $t=[1, \cdots, T]$ and $T=128$.

First, we show the simulation in a single-sensor case, where the input signal-to-noise ratio (SNR) is $10 \mathrm{~dB}$, and $60 \%$ of the data samples are randomly missing. Fig. 1(a) shows the realpart waveform with the missing samples marked in red, and its corresponding Wigner-Ville distribution (WVD) is shown in Fig. 1(b). The effect of spreading artifacts is clearly observed in the WVD. To improve the TF domain representation, we apply the AOK. The corresponding TFD, with enhanced signal auto-terms and reduced artifacts, is shown in Fig. 1(c). We have also included the TFSR reconstructed using OMP with

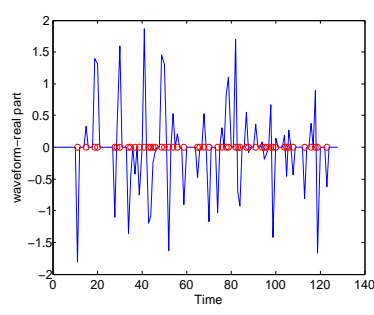

(a) real-part waveform

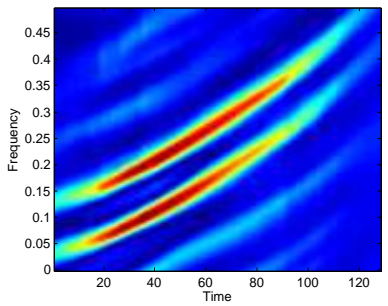

(c) TFD

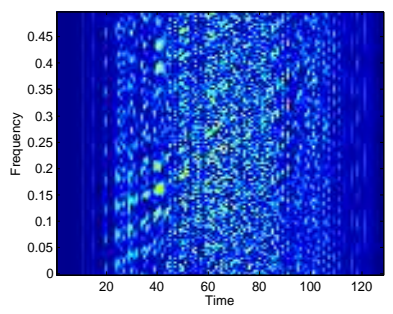

(b) WVD

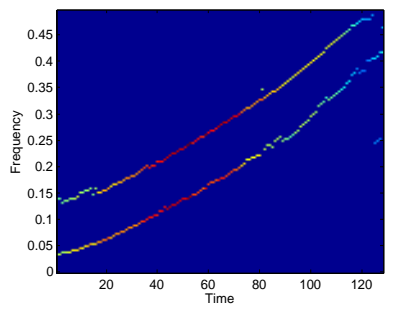

(d) TFSR from OMP
Fig. 1. Waveform and transformed domain representations of a twocomponent FM signal with $60 \%$ of missing samples (single sensor, $\mathrm{SNR}=10 \mathrm{~dB}$ ).
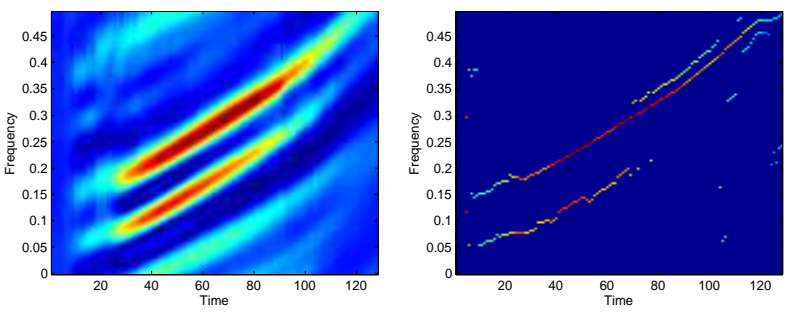

(a) TFD and TFSR from a single sensor
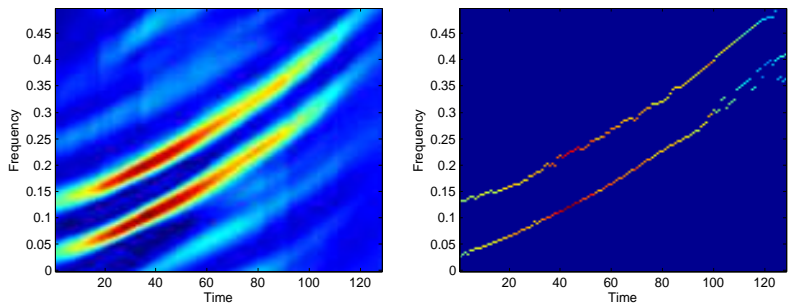

(b) TFD and TFSR from four sensors in the same missing pattern
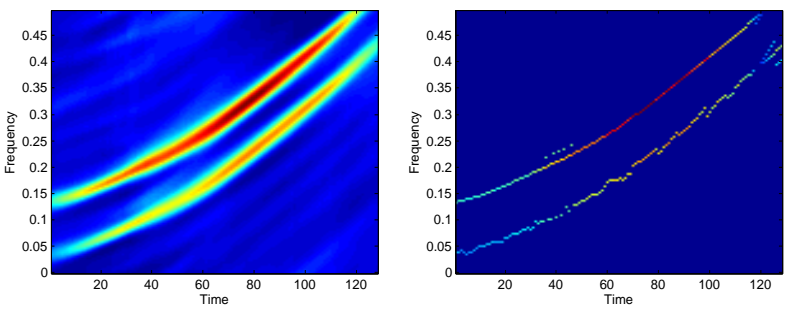

(c) TFD from four sensors in the different missing pattern

Fig. 2. TFD and TFSR of a two-component FM signal with $60 \%$ of missing samples $(\mathrm{SNR}=4 \mathrm{~dB})$.

the true instantaneous sparsity in Fig. 1(d). The reconstructed TFSR closely follow the auto-term signatures. 

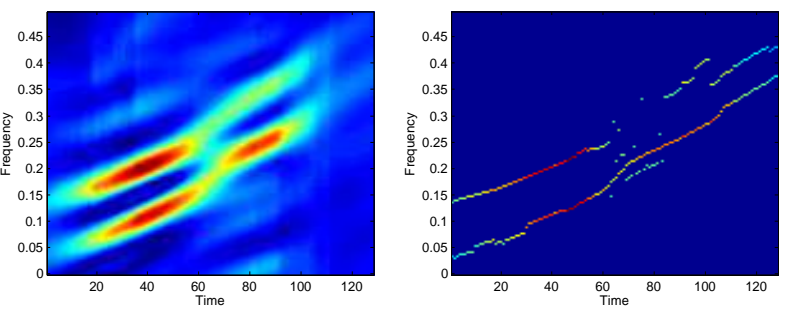

(a) TFD and TFSR from a single sensor
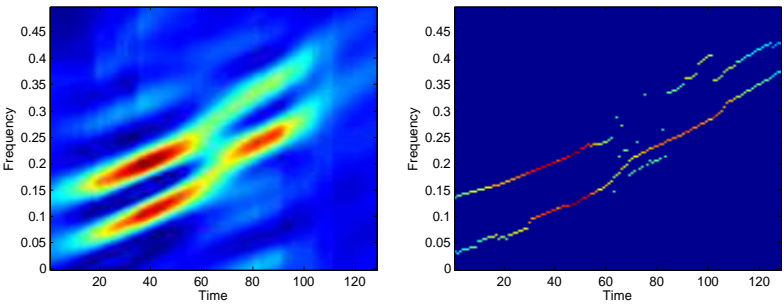

(b) TFD and TFSR from four sensors in the same missing pattern
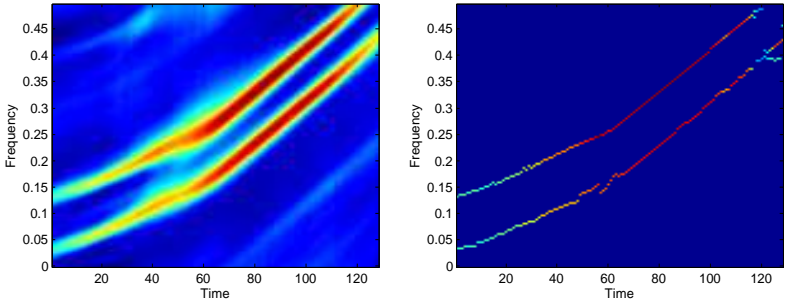

(c) TFD and TFSR from four sensors in different missing pattern

Fig. 3. TFD and TFSR of a two-component FM signal with $70 \%$ of missing samples $(\mathrm{SNR}=10 \mathrm{~dB})$.

In the next two examples, we respectively consider the effect of reduced SNR and increased number of missing samples, and examine the capability of multi-sensor platform. Two different situations are considered for the multi-sensor case, i.e., when the missing pattern is the same for all the sensors, and when different missing patterns are used.

\section{A. Effect of Reduced SNR}

In this example, we consider the same scenario as in Fig. 1 but the SNR is reduced to $4 \mathrm{~dB}$. The AOK-kernelled TFD and the reconstructed TFSR from OMP, as depicted in Fig. 2(a), clearly show performance degradation as compared to the high SNR case as in Fig. 1. When we use four sensors with the proposed multi-sensor AOK, on the other hand, both TFD and OMP-based TFSR are significantly improved. It is evident from Fig. 2(b), for the case with identical missing pattern, and from Fig. 2(c), for the case with different random missing patterns over the different sensors, that both cases achieve an array again from the four sensors, rendering the TFD and TFSR performance comparable to Figs. 1(c) and 1(d) for the single-sensor measurement with a $10 \mathrm{~dB}$ input SNR.

\section{B. Effect of Higher Number of Missing Samples}

In the next example, we keep the input SNR at $10 \mathrm{~dB}$ but increase the number of missing samples to $70 \%$. Fig. 3(a) shows poor single-sensor TFD and the corresponding TFSR obtained from OMP.

In Fig. 3(b), we show the TFD and the corresponding TFSR reconstructed from the four sensors with an identical sampling pattern used in all the sensors. The yielding TFD and TFSR results are very similar to the single-sensor case because it is a high SNR case, and the array observations with the same missing pattern do not improve the effect due to a high number of missing samples. On the other hand, as shown in Figs. 3(c), the TFSR is reliably constructed in the four-sensor case when different sampling patterns are used in each sensor. This example clearly demonstrates the importance of exploiting different sampling patterns in each sensor when the number of missing samples is high.

\section{CONCLUSION}

Compressive sensing (CS) techniques enable effective time-frequency (TF) signature reconstruction from randomly thinned nonstationary signal samples by exploiting the sparsity of such signals when viewed in the TF domain. While CSbased TF approaches were considered for a single-sensor scenario, we extend such treatment into a multi-sensor platform. In particular, we considered in this paper the design of datadependent adaptive kernel, from the combined observation at all sensors. This enables effective mitigation of undesired components, including artifacts due to missing data, crossterms, and noise. We have presented a number of examples to demonstrate the effectiveness of the multi-sensor TF signature reconstruction with the proposed kernel design. It was emphasized that the sensors must use different sampling patterns to combat the effect of missing samples in TF signature representation.

\section{REFERENCES}

[1] L. Cohen, Time-Frequency Analysis: Theory and Applications. Prentic-Hall, 1995.

[2] M. Akay, Time Frequency and Wavelets in Biomedical Signal Processing. IEEE Press, 1997.

[3] M. Skolnik, Radar Systems. McGraw-Hill, 2001.

[4] V. C. Chen and H. Ling, Time-Frequency Transforms for Radar Imaging and Signal Analysis. Artech House, 2002.

[5] B. Boashash, Time Frequency Signal Analysis and Processing. Elsevier, 2003.

[6] P. Flandrin, M. Amin, S. McLaughlin, and B. Torresani, "Special issue on time-frequency analysis and applications," IEEE Signal Proc. Mag., vol. 30, no. 6, 2013.

[7] Y. Zhang, W. Mu, and M. G. Amin, "Time-frequency maximum likelihood methods for direction finding," J. Franklin Inst., vol. 337, no. 4, pp. 483-497, 2000.

[8] Y. Zhang, W. Mu, and M. G. Amin, "Subspace analysis of spatial time-frequency distribution matrices," IEEE Trans. Signal Proc., vol. 49, no. 4, pp. 747-759, 2001.

[9] Y. D. Zhang and M. G. Amin, "Array processing for nonstationary interference suppression in DS/SS communications using subspace projection techniques," IEEE Trans. Signal Proc., vol. 49, no. 12, pp. 3005-3014, 2001.

[10] W. Mu, M. G. Amin, and Y. Zhang, "Bilinear signal synthesis in array processing," IEEE Trans. Signal Proc., vol. 51, no. 4, pp. $90-100,2003$.

[11] M. G. Amin, Y. Zhang, G. J. Frazer, and A. R. Lindsey, "Spatial time-frequency distributions: Theory and applications," in L. 
Debnath (ed.), Wavelets and Signal Processing, Birkhauser, 2003.

[12] Y. Zhang, B. A. Obeidat, and M. G. Amin, "Spatial polarimetric time-frequency distributions for direction-of-arrival estimations," IEEE Trans. Signal Proc., vol. 54, no. 4, pp. 13271340, 2006.

[13] Y. Zhang and M. G. Amin, "Blind separation of nonstationary sources based on spatial time-frequency distributions," EURASIP J. Applied Signal Proc., vol. 2006, article ID 64785, 13 pp., 2006.

[14] Y. D. Zhang, M. G. Amin, and B. Himed, "Joint DOD/DOA estimation in MIMO radar exploiting time-frequency representations," EURASIP J. Advances in Signal Proc., vol. 2012, no. 1, 2012:102, 2012.

[15] H. Choi and W. J. Williams, "Improved time-frequency representation of multicomponent signals using exponential kernels," IEEE Trans. Acoust., Speech, Signal Proc., vol. 37, no. 6, pp. 862-871, 1989.

[16] M. G. Amin, "Spectral decomposition of time-frequency distribution kernels," IEEE Trans. Signal Proc., vol. 42, no. 5, pp. 1156-1165, 1994.

[17] D. L. Jones and R. G. Baraniuk, "An adaptive optimal-kernel time-frequency representation," IEEE Trans. Signal Proc., vol. 43, no. 10, pp. 2361-2371, 1995.

[18] M. G. Amin and W. J. Williams, "High spectral resolution time-frequency distribution kernels," IEEE Trans. Signal Proc., vol. 46, no. 10, pp. 2796-2804, 1998

[19] B. Jokanovic, M. G. Amin, Y. D. Zhang, and F. Ahmad, "Adaptive time-frequency kernel design for sparse joint-variable signal representations," in Proc. European Signal Proc. Conf., Lisbon, Portugal, Sept. 2014.
[20] L. Stankovic, S. Stankovic, I. Orovic, and Y. D. Zhang, "Timefrequency analysis of micro-Doppler signals based on compressive sensing," in M. G. Amin (ed.), Compressive Sensing for Urban Radars, CRC Press, 2014.

[21] Y. D. Zhang, M. G. Amin, and B. Himed, "Reduced interference time-frequency representations and sparse reconstruction of undersampled data," in Proc. European Signal Proc. Conf., Marrakech, Morocco, Sept. 2013.

[22] Y. D. Zhang and M. G. Amin, "Compressive sensing in nonstationary array processing using bilinear transforms," in Proc. IEEE Sensor Array and Multichannel Signal Proc. Workshop, Hoboken, NJ, Jun. 2012.

[23] P. Flandrin and P. Borgnat, "Time-frequency energy distributions meet compressed sensing," IEEE Trans. Signal Proc., vol. 58, no. 6, pp. 2974-2982, 2010.

[24] Q. Wu, Y. D. Zhang, and M. G. Amin, "Continuous structure based bayesian compressive sensing for sparse reconstruction of time-frequency distribution," in Proc. Int. Conf. Digital Signal Proc., Hong Kong, China, Aug. 2014.

[25] J. A. Tropp and A. C. Gilbert, "Signal recovery from partial information via orthogonal matching pursuit," IEEE Trans. Info. Theory, vol. 53, no. 12, pp. 4655-4666, 2007.

[26] R. Tibshirani, "Regression shrinkage and selection via the lasso," J. Royal Statistical Society, Series B, vol. 58, no. 1, pp. 267-288, 1996.

[27] S. Ji, D. Dunson, and L. Carin, "Multitask compressive sampling," IEEE Trans. Signal Proc., vol. 57, no. 1, pp. 92-106, 2009.

[28] Q. Wu, Y. D. Zhang, M. G. Amin, and B. Himed, "Complex multitask Bayesian compressive sensing," in Proc. IEEE ICASSP, Florence, Italy, May 2014. 